\title{
Adaptive concept of state estimation with incomplete state vector measurement
}

\author{
Tadeusz Kwater ${ }^{{ }^{*}}$, Pawet Krutys ${ }^{1}$, Robert Pękala ${ }^{2}$, Bogdan Kwiatkowski ${ }^{1}$ \\ ${ }^{1}$ University of Rzeszow, Faculty of Mathematics and Natural Sciences, Department of Computer \\ Engineering \\ ${ }^{2}$ The State Higher School of Technology and Economics in Jaroslaw
}

\begin{abstract}
The paper presents the design and simulation experiments of the adaptive approach in the estimation of the object state realized by filter whose gain is calculated on-line. The adopted concept of determining the gain uses a defined for this purpose signal called an error and on the basis of its waveform features introduces an incremental correction of the amplification factor of the estimation filter. The obtained results of state estimation are characterized by stability and strong correctness even for cases of non-stationary disturbances
\end{abstract}

\section{Introduction}

Presently in engineering due to the contemporary requirements formulated to increase research and development of methods available makes it dominate the so-called "soft tasks", the essence of which are: probabilistic methods, approximate sets, uncertain variables, fuzzy logic, neural networks and adaptive algorithms. They provide a highly differentiated formal apparatus, especially as these methods can be combined. It seeks to apply first of all specialized computer techniques, thanks to which processes of automation of measurement and globalization of information systems are more effective [1]. Approaches associated to adaptation strategies can lead to sequential Monte Carlo algorithms also called molecular filter methods $[1,2,3]$. This is connected to additional requirements in terms of computing power. Many issues related to object recognition are solved using ANN-learning. In such cases there is a need for a learning process usually associated with data preprocessing $[4,5,6]$. The article proposes the construction of an algorithm with an adaptive approach to state estimation by a filter (similar to the Kalman filter), in which the gain coefficient is modified depending on the some characteristic features of the error signal defined for this purpose. Simulation studies were conducted for a biochemically contaminated river described by partial differential equations. When interpreting the description of these phenomena along the so-called characteristics, a set of ordinary differential equations was obtained [7]. This approach ensures unchanged accuracy of the description of biochemical phenomena in the river. For a thorough assessment of estimation process is employed the Root Mean Square Error (RMSE) similar as were used in $[8,9]$.

\section{Mathematical model of the polluted river}

Two indicators, ie biochemical oxygen demand (BOD) and demend oxygen (DO), were used to describe river water quality. Model equations were obtained by considering the

\footnotetext{
*Corresponding author: tkwater@ur.edu.pl
} 
mass balance of oxygen. For a better interpretation of large lateral tributaries, the river was divided into sections between these tributaries.

The mathematical model BOD-DO for the part of the river considered by the i-th section of the river takes on the form $[7,10-11]$ :

$$
\frac{d}{d t} x_{i}+\frac{d}{d z} V x_{i}=A x_{i}+B w
$$

with boundary conditions:

$$
x(0, t), x\left(z, t_{0}\right)
$$

where: $x(x, t)=\operatorname{col}\left[x_{1}, x_{2}\right]$ - vector representing respectively $\mathrm{BOD}$ and $\mathrm{DO}, w_{r}(z, t)$ vector representing the noise of a distributed, $V=\left[\begin{array}{ll}v & 0 \\ 0 & v\end{array}\right]$ - diagonal matrix with flow velocities $v, A=\left[\begin{array}{cc}-k_{1} & 0 \\ -k_{2} & -k_{3}\end{array}\right]$ - a state matrix in which the coefficients $k_{i}$ of the selfpurification rate of water, $B=\left[\begin{array}{ll}1 & 0 \\ 0 & 1\end{array}\right]$ - matrix influence of the disturbances, $w=\operatorname{col}\left[\begin{array}{ll}w_{1} & w_{2}\end{array}\right]$ - Vector of influenced of the local sources of oxygen from photosynthesis, oxygen consumption by bottom sediments or BOD and DO small inflows $[10,11]$.

The boundary conditions included in the model are described by initial and boundary conditions.

Initial condition - refers to the whole length of the segment at the moment $t=t_{0}$

$$
x_{i}\left(z, t_{0}\right)=x_{i 0}(z)
$$

Boundary condition - refers to the beginning of the section under consideration in the time domain:

$$
x_{i}(0, t)=M_{i} x_{i-1}(1, t)+w_{b i}(t)+R_{b i} u_{b i}(t)
$$

Another way of interpreting the process of river purification is to convert partial differential equations into many ordinary differential equations along the so-called characteristics. It is based on the interpretation of the description of water pollution in the case when BOD and RT index values are observed for a freely moving volume of water. Then the quality indicators depend only on time. For the $i$-th section of the river the characteristics are defined by the dependence:

$$
\frac{d}{d t} z_{i}(t)=v_{i}\left(z_{i}(t)\right)
$$

whose boundary points satisfy the condition:

$$
\int_{t_{0, i}}^{t_{0, i+1}} v_{i}\left(z_{i}(t)\right) d t+z_{o i}=1
$$

where $z_{0 i} \in[0,1]$ It is the coordinate of the initial point $t_{01}$. 
It was assumed that the measurement is carried out only one coordinate state. Selection is based on ease of implementation measurements and concerns the $\mathrm{x}_{2}$ (DO). The resignation of $x_{1}$ (BOD) measurements results from the need for long-term laboratory service, which makes them useless, for exemple in the control process. So the measurement equation is as follows:

$$
y(t)=C x_{2}(t)+v_{p}(t)
$$

where: $C=[0,1]-$ this is a measurement matrix, $v_{p}(t)-$ This is a measurement distortion with Gaussian distribution.

The state of polluted river on the characteristics described by ordinary differential equations receiving form:

$$
\frac{d}{d t} x\left(z_{i}(t), t\right)=A\left(z_{i}(t)\right) x\left(z_{i}(t), t\right)+B w_{r}\left(x_{i}(t), t\right)
$$

where the initial condition takes the form: $x\left(z_{i}\left(t_{0}\right), t_{0}\right)=x_{0}\left(z_{i 0}\right), i=1,2,3, \ldots$

$\mathrm{B}$ - matrix influence of the disturbances, and $w_{r}$ is the signal noise of disturbance. For the object described by equations (7) and measurements (6), we formulate the problem of estimation [11 - 13].

\section{The process estimation of state by the filter}

Approximation of actual description of phenomena by means of mathematical modeling is not an easy task. Among the many solutions to, the following: a mathematical model of the stochastic signals are added to the appropriate distribution. They represent the variability of external factors. The main task of estimation in such cases is to obtain the best possible information about the system based on measurements. A good solution is to use the Kalman filter. In this class of linear estimators, it provides a minimum mean square error of estimation. In practice, it may happen that the measurement does not include all coordinates of the state vector. This means that not all elements of the state vector are used to reproduce the full state vector $[11,14-16]$. This effect is especially useful in control systems where missing system information can be obtained from disturbed and indirect measurements. In general, the Kalman filter is a recursive algorithm for estimating the state vector model of a dynamic system using measurements of this system.

The Kalman filter equation is given by the following differential equation:

$$
\hat{x}(t)=A \hat{x}(t)+K_{F}(t)(y(t)-C \hat{x}(t)), \hat{x}\left(t_{0}\right)
$$

where: $\mathrm{y}-$ measurements, and $\mathrm{K}_{\mathrm{F}}-$ the gain coefiction of Kalman filter:

$$
K_{F}(t)=\tilde{P}(t) C^{T} R^{-1}
$$

in which $\widetilde{\mathrm{P}}(\mathrm{t}), \mathrm{C}, \mathrm{R}$ are, respectively, the estimation error covariance matrix, the measurement matrix and the covariance of measurement disturbances from equation (6).

In order to determine $\mathrm{K}_{\mathrm{F}}(\mathrm{t})$ the matrix $\widetilde{\mathrm{P}}(\mathrm{t})$ representing the covariance of the estimation error should be known we need to know this matrix. It fulfills the following Riccati's differential equation:

$$
\frac{d \tilde{P}}{d t}=A \tilde{P}(t)+\tilde{P}(t) A^{T}-\tilde{P}(t) C^{T} R^{-1} C \tilde{P}(t)+L Q L^{T}, t \geq t_{0},
$$

with initial condition,

$$
\widetilde{O}\left(s_{/}\right)<O^{\prime} s /(
$$

Finally, to determine the state estimate is necessary to solve two equations, ie. A filter equation and Riccati equation. The quality of the estimate obtained from equation (11) depends to a large extent on the filter gain $\mathrm{K}_{\mathrm{F}}(\mathrm{t})$. Its value depends on the characteristics of the stochastic signals affecting the given data object in the form of covariance of system 
and measurement disturbances. Determining the covariance of disturbance signals is sometimes a difficult task. Therefore, other solutions to eliminate these obstacles are being sought. One of the proposals is an approach in which the filter gain coefficient adaptively adjusts.

\section{Adaptive modification of the filter gain}

In the proposed filter based on measurements recursively determines the estimate of the state vector of the object and modification gain filter. It should be noted that both the object and the measurements are subjected to disturbaced signals. This approach avoids the inconvenience of having to estimate the characteristics of the signals affecting the object under consideration. They can be much differentiated. At Kalman's approach, nonlinear differential equations are solved to determine the filter gain coefficient. The essence of the proposed approach is the application of the difference between the measurement and the corresponding co-ordinate state estimate. This defined signal will be used to correct the value of the gain coefficient. This correction is made for each measurement moment with a suitable precaution coefficient. With these assumptions, the constructed filter determines the estimation values based on the measurements and the actual gain KF. The value of the filter gain correction it comes from the minimization error of the estimation, which results in the "adaptation" to the current conditions of the object's functioning. The algorithmic diagram of the algorithm is presented in Fig. 1.

The definition of an adaptive error is defined by the following relationship:

$$
\varepsilon(t)=y(t)-\hat{x}_{2}(\mathrm{t})
$$

where: $y(t)$ - the measurement at time $\mathrm{t}$, and $\hat{x}_{2}(\mathrm{t})$ - estimate indicator of DO.

An error derivative is also used for the gain correction calculation, which assumes the following form:

$$
\frac{d \varepsilon\left(t_{2}\right)}{d t}=\left(\varepsilon\left(t_{2}\right)-\varepsilon\left(t_{1}\right)\right) / d t, t_{2}>t_{1}
$$

The change in gain coefficients was assumed to be incremental, as did the change in weights in neuronal networks, according to the following formula:

$$
K_{F_{N}}=K_{F_{S}}+\eta \cdot d K_{F}
$$

where: $K_{F_{N}}$ - value of the filter gain factor after adjustment, $d K_{F}$ - correction of filter gain increase, $K_{F_{S}}$ - Initial value of the filter gain coefficient, $\eta=(0 \ldots .1)-$ Coefficient of change precaution .

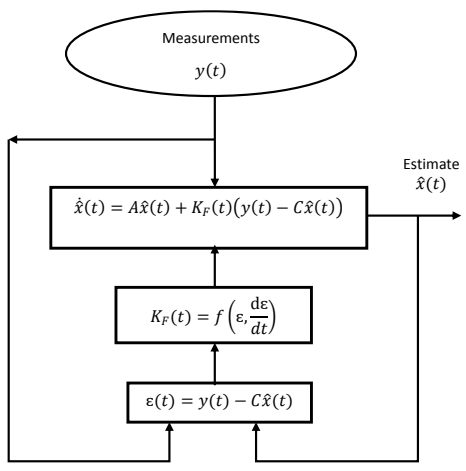

Fig. 1. Block diagram of adaptive estimation of state. 
Calculation of the increase of gain filter dK_F taken as a proportional function or in the form of tabulated and made dependent on the threshold value of the error $\varepsilon$, and the operation of addition or subtraction of the gain is carried out depending on the sign of the derivative of error. The implementation of this task is shown in the block diagram in Figure 2 . In the case of large errors of the $\varepsilon$, the value of precaution $\eta$ is smaller, which affects the stability of the algorithm. The values of $\eta$ in the algorithm were chosen using the experimental method. Stabilization and improvement of the quality of the adaptive algorithm was also obtained by taking into account several previous measurements relative to the current one. The change in the error value from the previous step significantly affected the change in the filter gain coefficient. Stabilization and improvement of the quality of the adaptive algorithm was also obtained by taking into account several previous measurements relative to the current one. The change in the error value from the previous step significantly affected the change in the filter gain coefficient.

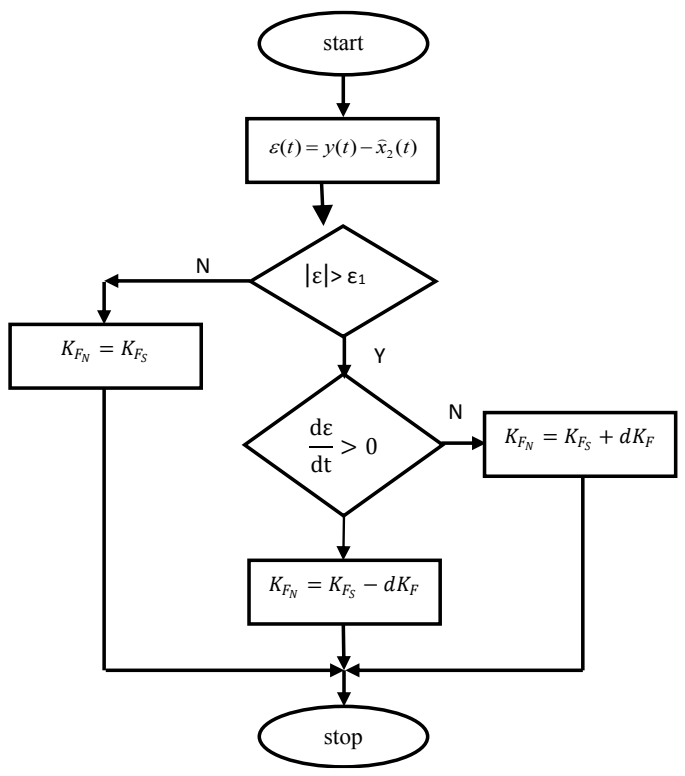

Fig. 2. Block diagram for calculating increment of filter gain coefficient changes.

The global block diagram of the adaptive algorithm is shown in Figure 3. It is worth noting that Block A is a schematic in which the conditions for changes in the gain factor of a filter are determined based on the value of errors and their derivatives in the so-called observation window. 


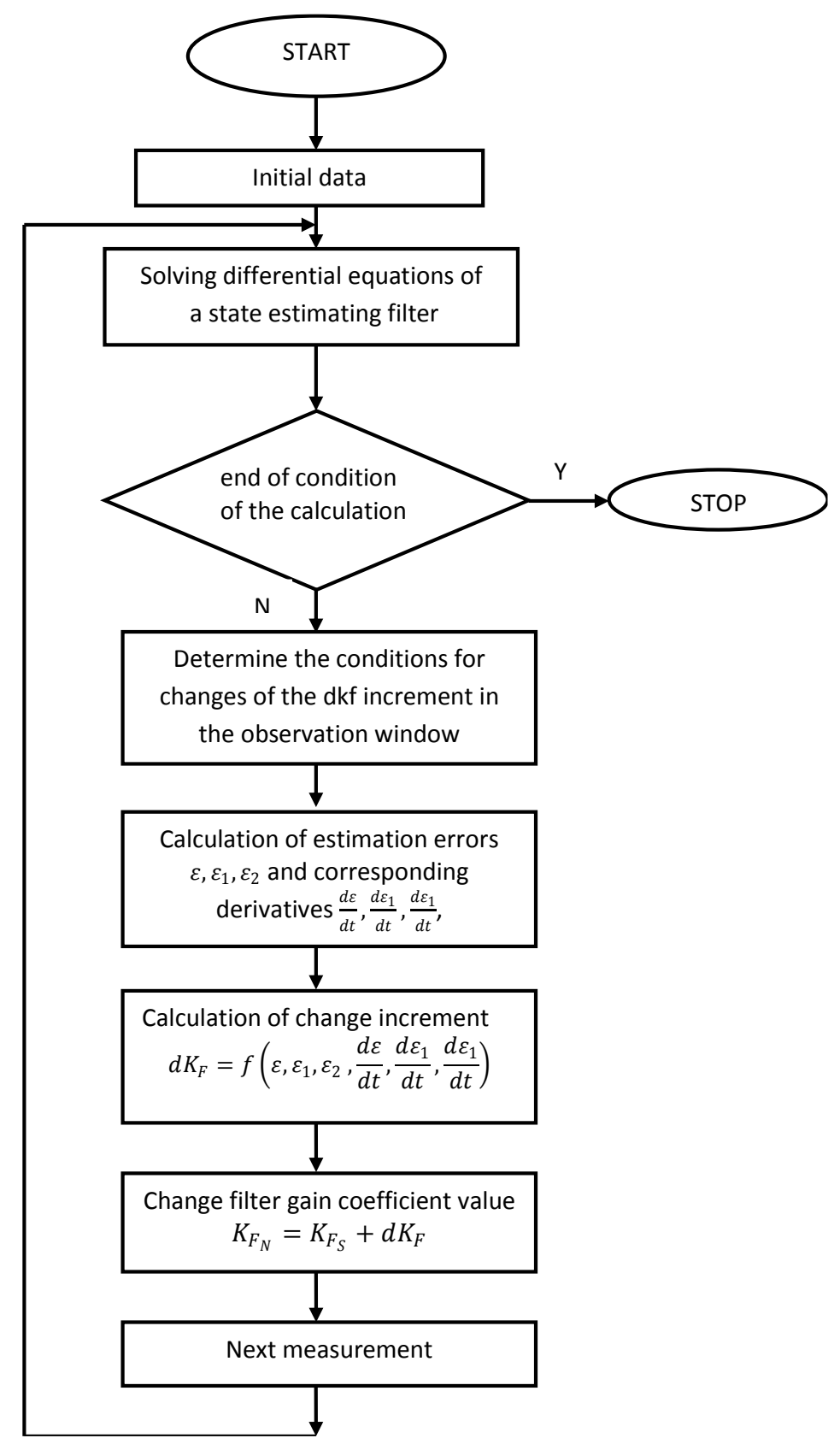

Fig. 3. Schematic flow diagram of an adaptive state estimation

The conducted simulation experiments were made for different values of configuration parameters occurring in the algorithm. As a measure of the quality of estimation accepted the square root of the integral squared estimation error, respectively for BOD and RT expressed formulas:

$$
W S B=\sqrt{\frac{1}{T} \int_{0}^{T} \varepsilon_{1}^{2} d t}
$$




$$
W S B=\sqrt{\frac{1}{T} \int_{0}^{T} \varepsilon_{2}^{2} d t}
$$

In which $\varepsilon_{1}(t)=x_{1}(t)-\hat{x}_{1}(t), \varepsilon_{2}(t)=x_{2}(t)-\hat{x}_{2}(t)$ and $x_{1}, \hat{x}_{1}$ is the coordinate of the BOD state and its estimate, while $x_{2}, \hat{x}_{2}$ - is the DO coordinate and its estimate.

\section{The simulation results}

In simulation studies, many algorithms were changed. In each case, stable solutions were obtained, ie estimations followed the changes in the state vector.

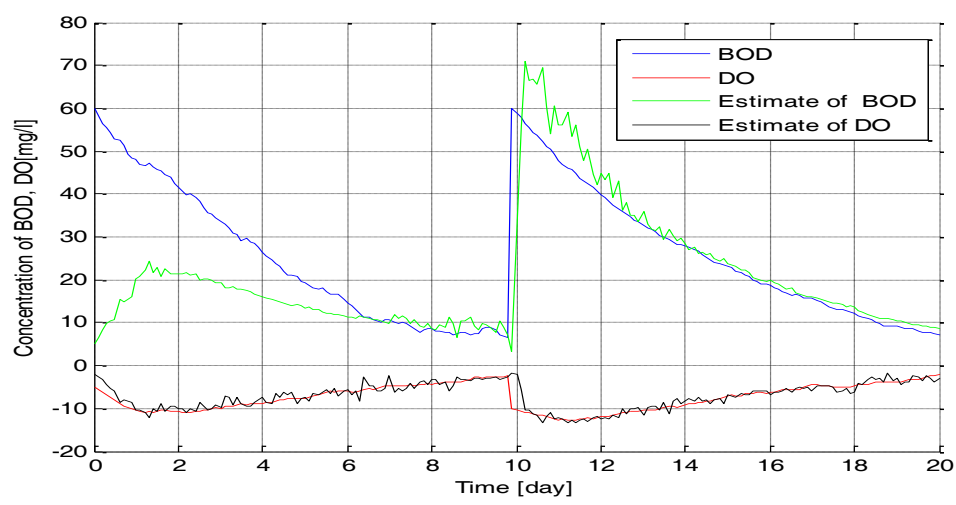

Fig. 4. BOD and DO changes and their estimation.

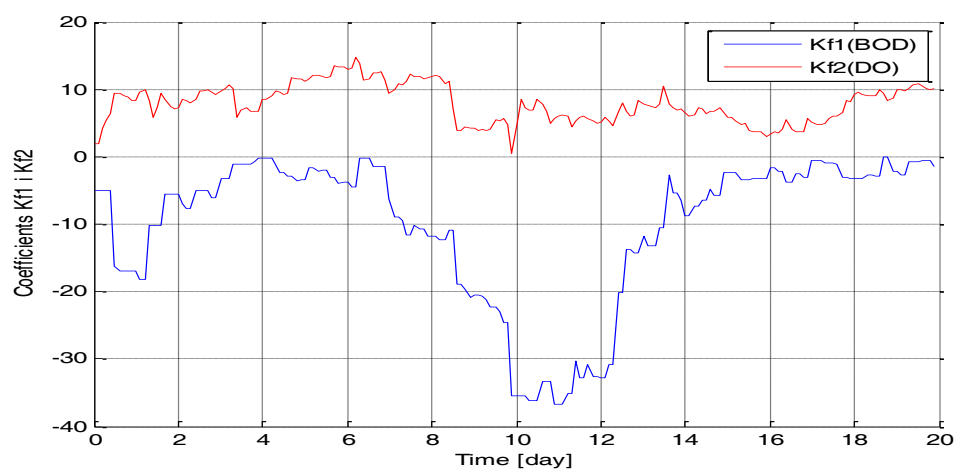

Fig. 5. Filter gain coefficients. 


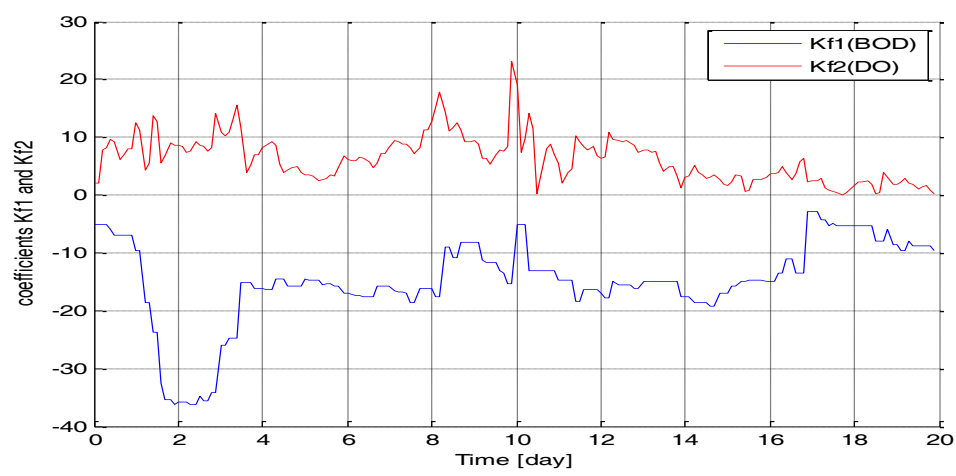

Fig. 6. Filter gain coefficients.

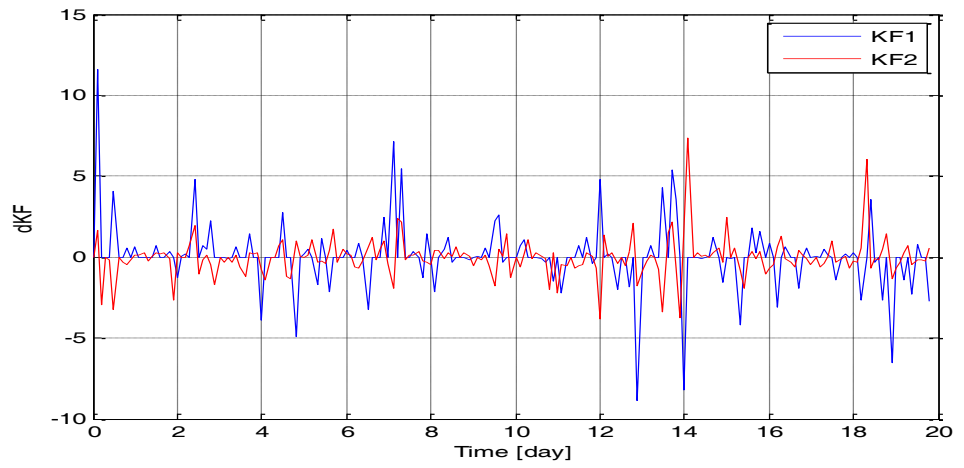

Fig. 7. Changes in gain coefficients.

In numerous simulation experiments, the intensity of measurement and system disturbances was changed. The sample results of the estimation quality tests are presented in Table 1 . In all cases, the correctness was obtained, which consisted in the fact that the BOD (15.a) rates always took much higher values than the ratios for RT (15.b).

Table 1. Values of estimation quality indicators.

\begin{tabular}{|l|l|l|l|l|l|}
\hline $\mathrm{Nr}$ & $\begin{array}{c}\text { Standard } \\
\text { deviation } \\
\text { for the } \\
\text { BOD ratio }\end{array}$ & $\begin{array}{c}\text { Standard } \\
\text { deviation } \\
\text { for the DO } \\
\text { ratio }\end{array}$ & $\begin{array}{c}\text { Standard } \\
\text { deviation of } \\
\text { the } \\
\text { measurement }\end{array}$ & $\begin{array}{c}\text { Estimation } \\
\text { quality } \\
\text { indicator for } \\
\text { BOD }\end{array}$ & $\begin{array}{c}\text { Estimation } \\
\text { quality } \\
\text { indicator for } \\
\text { DO }\end{array}$ \\
\hline 1 & 5.0 & 2.0 & 1.0 & 13.5 & 1.5 \\
\hline 2 & 3.0 & 4.0 & 2.0 & 13.5 & 1.75 \\
\hline 3 & 10.0 & 5.0 & 2.0 & 17.5 & 1.3 \\
\hline 4 & 5.5 & 2.5 & 1.0 & 14.5 & 1.0 \\
\hline 5 & 5.0 & 2.0 & 1.0 & 12.5 & 1.2 \\
\hline
\end{tabular}

This is due to the nature of the process being carried out, for which only the coordinate RT is measured. This means that information from the measurement of the BOD are small hence the great value of the index for the estimation of the BOD. The slight differences between positions 1 and 5 for the estimation indices (columns 5 and 6) result from a different implementation of the generated disturbances. 


\section{Summary}

The article proposes an algorithm to estimate the state in the form of a filter similar to the Kalman filter with adaptive selection of its gain. Its operation was realized using a defined error as the difference between the measurement and the corresponding indicator. It should be emphasized that the assumptions do not include all the coordinates of the state vector. In order to increase its flexibility and precision of operation, measurement window taking into account several previous measurements. The conducted simulation experiments for a hypothetical river with a length of 400 kilometers of polluted tributary in the middle of its length. The solution of differential equations was implemented by the Runge-Kutta algorithm, fourth order. For all cases, satisfactory results have been obtained, ie, the time course of the estimate and the coordinates of the state have always been consistent. The research involved changing the various parameters of the adaptive algorithm. Sudden large changes in BOD can be regarded as polluted large side inlets or as a non-stationary processes. For such cases, satisfactory results have been obtained. It turned out that the different initial values of the gain factor (if they are from the same range) did not have a significant effect on the results of the estimation of the state. The characteristic of the obtained estimation is the better quality for the estimation for which the measurements are made. The filter, despite the lack of BOD information (no measurements) generated its estimate of the correct trend of change. The implementation of the algorithm does not require high computing Power.

\section{References}

1. Kacprzyk J., Hryniewicz O., Kulczycki P. Techniki informacyjne w badaniach systemowych. WNT, Warszawa 2007.

2. Cornebise J., E Moulines, J Olsson, Adaptive refueling in particle filter algorithms, Workshop New directions in Monte Carlo Methods (2007), Fleurance, 25-29

3. Cornebise J., Moulines É., Olsson J., Adaptive methods for sequential importance sampling with application to state space models, 2008.

4. Farhang-Boroujeny B., ,Adaptive Filters. Theory and Applications\|, Wiley, 1998.

5. Kasprzak W., Adaptive computation methods in digital image sequence analysis. Warsaw University of Technology, Warszawa, No. 127, 2000

6. E. Dudek-Dyduch, Z. Gomolka, Neural network design without learning, Neural Networks and Soft Computing Book Series: Advances in Soft Computing, pp: 179187, (2003)

7. Chapra Steven C., Surface water-quality modeling, Waveland Press Inc., 2008

8. Z. Gomolka, B. Twarog, J. Bartman, Improvement of image processing by using homogeneous neural networks with fractional derivatives theorem, Discrete and Continuous Dynamical Systems- Series AI, ssue SUPPL., Pages 505-514, (2011)

9. Z. Gomolka, Neurons' Transfer Function Modeling with the Use of Fractional Derivative. DepCoS-RELCOMEX 2018., vol 761. (2019)

10. Kwater T., Krutys P.: Estimation of pollution of the river by artificial neural networks, Symbiosis of Engineering and Computer Science Wydawnictwo Uniwersytetu Rzeszowskiego, Rzeszów 2010, ISBN 978-83-7338-620-4, pp.37-66

11. Z.Gomolka, B.Twarog, E. Zeslawska, A. Lewicki, T. Kwater, Using Artificial Neural Networks to Solve the Problem Represented by BOD and DO Indicators, Water 2018, 10(1), 4; https://doi.org/10.3390/w10010004 
12. Pooran Singh Mahar, Bithin Datta, Optimal Identification of Ground-Water Pollution Sources and Parameter Estimation, Journal of Water Resources Planning and Management, Vol. 127, Issue 1 (February 2001)

13. Fox D., Adapting the sample size in particle filters through KLD-sampling Res. 22(11), 2008.

14. Haykin S.: Adaptive Filter Theory. Englewood Cliffs, New York: Prentice-Hall, 1991.

15. Singh M. G., Dynamical hierarchical control. North-Holland, Amsterdam 1977.

16. Z. Gomolka, B. Twarog, E. Zeslawska, Cognitive Investigation on Pilot Attention During Take-Offs and Landings Using Flight Simulator. Artificial Intelligence and Soft Computing., vol 10246., (2017) 